\title{
Application of an electronic wave-packet formalism to local-operator equations of motion for semiconductor lasers
}

\author{
Kerry Vahala and Amnon Yariv \\ California Institute of Technology, Pasadena, California 91125
}

(Received 3 July 1984)

\begin{abstract}
We develop a quantum mechanical formalism which facilitates the study of interactions locally in semiconductors. It is based on the operators $\psi^{\dagger}(m, \mathbf{x}, \mathbf{k}, t)$ and $\psi(m, \mathbf{x}, \mathbf{k}, t)$ which are defined so as to create and destroy electronic wave-packet states having well-defined position $\mathbf{x}$ and crystal momentum $\hbar \mathbf{k}$ in a given energy band $m$ of a crystal. We apply the formalism to develop local-operator equations of motion for semiconductor lasers.
\end{abstract}

\section{INTRODUCTION}

In studying lasers, it is often necessary to consider interactions between the lasing modes and the active medium locally (i.e., local material equations of motion) rather than in a spatially averaged fashion. Examples of this can be found in a myriad of topics which include intermodal beating, spatial hole burning, and diffusion damping of relaxation oscillations. In most laser systems the development of local equations of motion to study these effects is greatly facilitated by the atomic nature of the active medium. In such cases the individual components of the system, by their spatial smallness, conveniently sample the electron-radiation interaction locally and lead naturally to local rate equations. Exhaustive treatments by Lamb ${ }^{1}$ and by Haken $^{2}$ can be cited which elegantly illustrate this approach. Unfortunately, there do exist laser systems which do not fall within the scope of these local treatments; these are systems having delocalized eigenstates. A very important example is the semiconductor-laser (SL) system in which the preferred state space for all quantum treatments to date has been the electronic Bloch-state space (see, for example, Refs. 3-6). This space leads to convenient selection rules for electron-radiation-induced transitions, but is an extremely awkward space to use for treating local phenomena.

Despite the difficulties involved in formulating a set of local rate equations for SL's from first principles, a large number of treatments do exist which have successfully accounted for many aspects of local phenomena in SL's. These seem to fall into two categories: those which directly apply rate equations derived for gas lasers to the SL case and thus assume forms a priori for electronradiation interaction terms in local rate equations; and those in which a classical electric field interacts with an active layer crystal sliced into small local systems, each quantized with its own Bloch-state space. ${ }^{7}$ The latter approach, albeit more rigorous than the first, is somewhat artificial and must still treat interactions between local cubical systems in a heuristic fashion. Even with the success of the above methods, one is led to wonder what phenomena are overlooked through their simplicity; a glaring example is their inability to account for quantum fluctuation phenomena.
One goal of this paper is then to derive from first principles a set of local quantum-mechanical equations of motion for a SL. To do this we will work in a space of electronic wave-packet states. These wave packets have well-defined position and crystal momentum, and are often used as a conceptual tool to justify the tenets of semiclassical solid-state theory in which electrons and holes are treated in a classical fashion through the use of concepts such as effective mass, crystal momentum, etc. ${ }^{8}$ In this treatment we will define operators which create and destroy electronic wave packets within semiclassical phase spaces associated with each energy band of the crystal. The operators so defined will serve as the dynamic variables characterizing the active medium. An exact treatment based on these operators offers no advantage over a treatment based on Bloch states. We will show, however, that for certain classes of quantum interaction potentials, the electron-radiation interaction being one, a perturbation expansion of both matrix elements and operator anticommutators is possible. The approximation here requires that the system Hamiltonian and the dynamic variables vary slowly in comparison to the extent of an electronic wave packet. (This approximation is satisfied by a variety of interaction potentials and is not restricted to electronic wave packets; recently, Glushko has employed the approximation in a treatment of excitonphonon interactions based on an exciton wave-packet state. ${ }^{9}$ ) We will derive operator equations of motion correct to first order in this approximation; higher-order corrections will also be discussed.

As a quantum analog of classical phase-space dynamics the electronic wave-packet approach is in direct competition with certain well established techniques in quantum kinetic theory. ${ }^{10-13}$ of these methods the wave-packet approach is most closely related to the method of second quantization in phase space introduced by Klimontovich. ${ }^{11}$ In that method the dynamic variables are quantum fields in phase space whose quantum averages are Wigner distribution functions. ${ }^{10,12}$ In the present method it will be seen that certain operator pairs can be interpreted as density operators whose quantum average resembles a first-order distribution function (higher-order functions are also possible by taking groups of operator pairs, but these will not be discussed). We intend to contrast these 
methods elsewhere. ${ }^{14}$ Very briefly, however, the method of second quantization in phase space has the advantage of leading naturally to representations which are diagonal in $x$ and $p$. The wave-packet approach, as a result of the overcompleteness of the electronic wave-packet states, suffers from representations which are diagonal only to first order in the approximation discussed above. In many cases this is a serious disadvantage. When the approximation can be invoked, however, the wave-packet approach can become the preferable method. In the case considered here of electronic motion in a crystal with coupling to the radiation field this is especially true. Under these circumstances the Bloch-like character of the electronic wave-packet states makes them approximate eigenstates of the crystal, thus simplifying treatment of both the electron-lattice interaction and the electron-radiation interaction. The second goal of this paper then is to serve as a pedagogic example of the application of this wavepacket formalism to the specific case of the electronradiation interaction.

\section{WAVE-PACKET OPERATORS AND THE SYSTEM HAMILTONIAN}

In this section we define the field operators of the material and the lasing optical modes, and then write the system Hamiltonian in terms of these operators. The field operators of this analysis distinguish it from previous quantum treatments of the SL, giving a local description of the active medium and thus facilitating the treatment of multimode effects as well as transport phenomena. To illustrate some of the difficulties normally encountered in modeling a multimode SL quantum mechanically (besides the obvious problems involving electronic transport) we will first review an approach frequently employed, this being a nonlocal description based on Bloch states. Following this discussion we introduce the field operators of this analysis and their associated anticommutation relations. These operators will be seen to create and destroy electronic wave packets having well-defined position and crystal momentum, in the same sense as the electronic wave packets which make up the semiclassical picture of the Bloch electron. Finally, we will derive anticommutation relations and a system Hamiltonian, both correct to first order in the approximation discussed above. During the course of the derivation the limitations of this approximation are discussed and it is also shown how higherorder corrections to the Hamiltonian can be calculated.

A common approach to a quantum description of the electronic system of a semiconductor is to assume a multielectron wave function given by a symmetrized product of single-electron wave functions; as, for instance, can be accomplished using a Slater determinant. Most often the single-electron states are taken as Bloch states $\varphi_{m}(\mathbf{j}, \mathbf{x})$ (i.e., band index $m$ and crystal momentum wave vector $\mathbf{j}$; spin is neglected throughout this treatment) which satisfy the single-electron Schrödinger equation,

$$
(T+V) \varphi_{m}(\mathbf{j}, \mathbf{x})=\epsilon_{m}(\mathbf{j}) \varphi_{m}(\mathbf{j}, \mathbf{x}),
$$

where $T$ is the kinetic energy operator and $V$ describes only the crystal potential (both operators in the $\mathbf{x}$ repre- sentation). Using the basis set of symmetrized Bloch functions, annihilation and creation operators for these electronic states are defined. These operators obey the fermion anticommutation relations,

$$
\begin{aligned}
& \left\{a_{m}(\mathbf{j}, t), a_{n}^{\dagger}(\mathbf{k}, t)\right\}=\delta_{m n} D(\mathbf{j}-\mathbf{k}), \\
& \left\{a_{m}(\mathbf{j}, t), a_{n}(\mathbf{k}, t)\right\}=0, \\
& \left\{a_{m}^{\dagger}(\mathbf{j}, t), a_{n}^{\dagger}(\mathbf{k}, t)\right\}=0,
\end{aligned}
$$

where $\{$,$\} represents the operation of anticommutation,$ $\delta_{m n}$ is the Kronecker delta, and $D(\mathbf{j}-\mathbf{k})$ is the Dirac delta function (i.e., we assume the crystal is large enough to justify treating the crystal momentum as a continuous quantity). The system Hamiltonian is then expressed in terms of these operators; a simple example being the unperturbed Bloch electron system,

$$
\begin{aligned}
H & =\sum_{m, n} \int d \mathbf{j} d \mathbf{k}\langle m \mathbf{j}|H| n \mathbf{k}\rangle a_{m}^{\dagger}(\mathbf{j}, t) a_{n}(\mathbf{k}, t) \\
& =\sum_{m} \int d \mathbf{j} \epsilon_{m}(\mathbf{j}) a_{m}^{\dagger}(\mathbf{j}, t) a_{m}(\mathbf{j}, t)
\end{aligned}
$$

which is the familiar sum of number operators over all states weighted by the energy of each state. Then, with Hamiltonian and anticommutation relations in hand, the Heisenberg equations of motion can be written for a particular set of self-consistent operators. The final step is to solve these equations of motion.

Several aspects of the above approach make it unsuitable for our purposes. First consider the electronradiation interaction matrix element,

$$
\left\langle m \mathbf{j}\left|H_{I}\right| n \mathbf{k}\right\rangle=\left\langle m \mathbf{j}\left|-\frac{e}{m} \mathbf{A}(\mathbf{r}, t) \cdot \mathbf{p}\right| n \mathbf{k}\right\rangle .
$$

In particular, the treatment of the vector potential $\mathbf{A}(\mathbf{r}, t)$ spatial dependence in this matrix element. For an atomic system this spatial dependence can be approximated by the vector potential's value at the atom's position (as given, say, by its nucleus), because the atomic wave states involved in the transition are highly localized in comparison to the scale of the optical wavelength. As such, the resulting matrix element takes on a unique spatial dependence characterized by the optical mode. It is this spatial dependence which leads to spatial hole burning of a gas of inverted atoms or molecules, and which is also of importance in multimode lasers where the spatial dependence of the induced polarization determines, in part, mode coupling. At the opposite extreme are the electronic Bloch states. These states are delocalized, having the wellknown translational property

$$
\varphi_{m}(\mathbf{j}, \mathbf{x}+\mathbf{R})=\varphi_{m}(\mathbf{j}, \mathbf{x}) e^{i \mathbf{j} \cdot \mathbf{R}},
$$

where $\mathbf{R}$ is a lattice translation vector. Using this property the matrix element (2.6) can be rewritten as follows:

$$
\begin{aligned}
\left\langle m \mathbf{j}\left|-\frac{e}{m} \mathbf{A}(\mathbf{r}) \cdot \mathbf{p}\right| n \mathbf{k}\right\rangle & \\
& \approx-\frac{e}{m} \sum_{\mathbf{R}} e^{i(\mathbf{k}-\mathbf{j}) \cdot \mathbf{R}} \mathbf{A}(\mathbf{R}) \cdot\langle m \mathbf{j}|\mathbf{p}| n \mathbf{k}\rangle_{\mathrm{pc}},
\end{aligned}
$$


where the integration now takes place over a primitive cell and, as with the atomic system, the slowly (over a unit cell) varying vector potential has been removed from the integral. This leaves only the evaluation of the sum over crystal translation vectors. Assuming $\mathbf{A}(\mathbf{r})$ represents a single longitudinal mode with wave vector $\mathbf{k}_{m}^{\prime}$, it is clear that the sum is strongly peaked for $\mathbf{k}-\mathbf{j}= \pm \mathbf{k}_{m}^{\prime}$, which is the selection rule describing a shift in crystal momentum caused by the photon emission or absorption. This shift is relatively small in comparison to the dimensions of the Brillouin zone and therefore it is standard practice to neglect it altogether, resulting in the " $k$-selection rule" for direct optical transitions in a semiconductor. Unfortunately, this rule altogether eliminates the spatial dependence of the optical mode and therefore precludes the study of multimode interactions and effects such as spatial hole burning. If the $\mathbf{k}_{m}^{\prime}$ dependence of the selection rule is retained, then the resulting equations become rather complicated, each mode coupling different pairs of states. It is also obvious that the inclusion of transport phenomena into the model is made very cumbersome by this approach. The aforementioned difficulties have at their root the delocalized nature of the chosen electronic state space.

An inherently local description of the electronic system, which successfully explains many aspects of electronic transport, is semiclassical solid-state theory. In this approach electrons and holes in a crystal are ascribed properties characteristic of their free classical counterparts (e.g., effective mass, momentum, position, etc.). Such notions greatly simplify the study of transport, but being to a certain extent classical they are either incapable of accounting for certain quantum interactions (e.g., interband transitions) or must be modified heuristically to do so; as, for instance, is done in writing a local carrier-density rate equation which includes electron-radiation stimulated recombination terms. From a quantum-mechanical viewpoint the semiclassical electron is a wave packet whose spatial extent is macroscopically small, but microscopically large enough to give the packet a well-defined crystal momentum. Such a wave packet would encompass several hundred lattice sites along a given direction, thus having a breadth in $k$ space much narrower than a Brillouin zone (see Fig. 1). We now explore the use of these quasiclassical wave packets as a basis set instead of Bloch states. By themselves these new states will not improve matters over an analysis based on Bloch states; in fact, they will appear at first to be a complication. Combined with an approximation, discussed later in the section, however, a significant simplification of the problem occurs. With the approximation, the wave-packet states allow us to extend the semiclassical picture of the electron to rigorously account for certain classes of perturbing potentials while maintaining quantum-mechanical consistency.

The Bloch states and their respective annihilation and creation operators will be used as a tool to develop the quasiclassical wave-packet states and their respective annihilation and creation operators. To begin we define a quasiclassical wave packet localized about $\mathbf{x}$ and $\mathbf{k}$ in the $m$ th band as
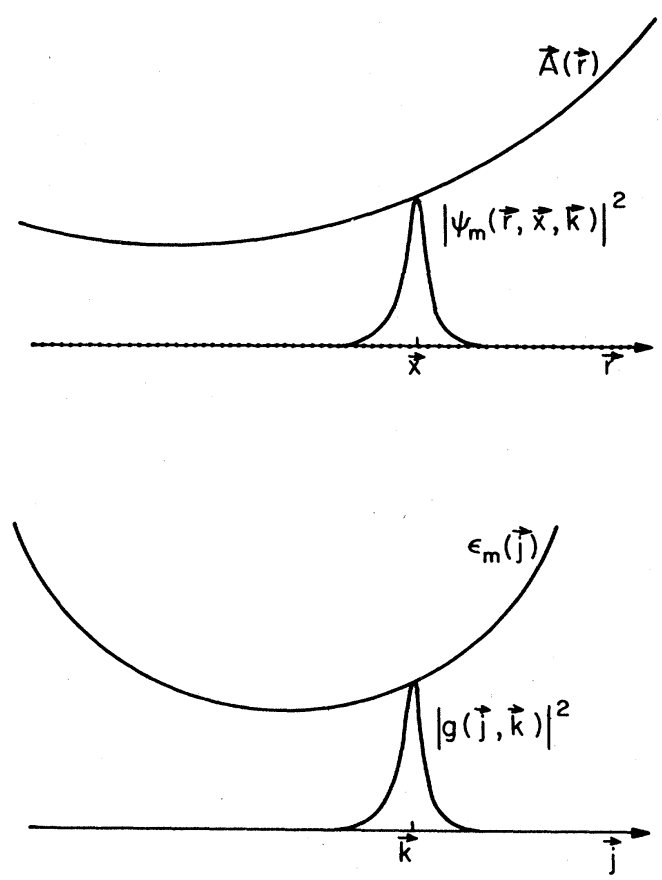

FIG. 1. Projection of a single-particle wave-packet state onto real $|\mathbf{r}\rangle$ space (upper plot) and Bloch space $|m, \mathbf{j}\rangle$ (lower plot). The wave packet is macroscopically small so that it effectively samples the vector potential $\mathbf{A}(\mathbf{r})$ locally, but is microscopically large enough to retain "Bloch" character and have a welldefined crystal momentum and energy.

$$
|\psi(m, \mathbf{x}, \mathbf{k})\rangle \equiv \int d \mathbf{j} g(\mathbf{j}, \mathbf{k}) e^{-i \mathbf{j} \cdot \mathbf{x}}|m \mathbf{j}\rangle
$$

where the integration is over the Brillouin zone and where $g(\mathbf{j}, \mathbf{k})$ is a crystal momentum weighting function localized on $\mathbf{k}$; the exact nature of this weighting function is unimportant for our purposes, so long as it leads to an electronic wave packet in the sense discussed above. In Fig. 1 we show the general shape of $|g(\mathbf{j}, \mathbf{k})|^{2}=|\langle m, \mathbf{j} \mid \psi(m, \mathbf{x}, \mathbf{k})\rangle|^{2}$ and $\left|\psi_{m}(\mathbf{r}, \mathbf{x}, \mathbf{k})\right|^{2}$ $\equiv|\langle\mathbf{r} \mid \psi(m, \mathbf{x}, \mathbf{k})\rangle|^{2}$. Both are localized functions in comparison to variations of the Hamiltonian which include variations of the unperturbed Bloch energy function $\epsilon_{m}(j)$ and variations of the perturbation energy brought about through the vector potential $\mathbf{A}(\mathbf{r})$. Expression (2.9) transforms our picture from one based on the variable $\mathbf{j}$ within a given band $m$ to one based on the variables $(\mathbf{x}, \mathbf{k})_{m}$ which represent the semiclassical "center-of-mass" position (in real and crystal wave-vector space) of a wave packet in band $m$. In this picture each band has associated with it a semiclassical phase space in which wave packets appear highly localized. Interband transitions represent particle exchanges between the phase spaces. It is straightforward to verify that the wave-packet states satisfy the completeness relation,

$$
\begin{gathered}
\sum_{m} \int d \mathbf{x} d \mathbf{k}|\psi(m, \mathbf{x}, \mathbf{k})\rangle\langle\psi(m, \mathbf{x}, \mathbf{k})| \\
=\sum_{m} \int d \mathbf{j}|m \mathbf{j}\rangle\langle m \mathbf{j}|=I
\end{gathered}
$$


provided

$$
(2 \pi)^{3} \int d \mathbf{k}|g(\mathbf{j}, \mathbf{k})|^{2}=1,
$$

where $I$ is the identity operator. As is often true for quasiclassical states in general, the wave-packet states are not orthogonal; from (2.9) we calculate

$$
\left\langle\psi(m, \mathbf{x}, \mathbf{k}) \mid \psi\left(n, \mathbf{x}^{\prime}, \mathbf{k}^{\prime}\right)\right\rangle=\delta_{m n} \int d \mathbf{j} g^{*}\left(\mathbf{j}, \mathbf{k}^{\prime}\right) g(\mathbf{j}, \mathbf{k}) e^{i \mathbf{j} \cdot\left(\mathbf{x}-\mathbf{x}^{\prime}\right)} .
$$

Thus, wave packets in different bands are orthogonal, but within a given band are not. It is obvious, however, that packets sufficiently well separated in a given phase space $(\mathbf{x}, \mathbf{k})_{m}$ are approximately orthogonal. As a specific case consider a weighting function $g(\mathbf{j}, \mathbf{k})$ with a Gaussian shape given by

$$
g(\mathbf{j}, \mathbf{k})=\frac{\sigma^{3 / 2}}{(2 \pi)^{9 / 4}} \exp \left[-\frac{\sigma^{2}}{4}|\mathbf{j}-\mathbf{k}|^{2}\right],
$$

where $\sigma$ has units of length in $x$ space and will be seen momentarily to give the approximate spatial breadth of a wave packet. Equation (2.13) has been normalized in accordance with (2.11) and when substituted into (2.12) yields

$$
\begin{aligned}
\left\langle\psi(m, \mathbf{x}, \mathbf{k}) \mid \psi\left(m, \mathbf{x}^{\prime}, \mathbf{k}^{\prime}\right)\right\rangle= & \frac{1}{(2 \pi)^{3}} \exp \left[\frac{i}{2}\left(\mathbf{k}+\mathbf{k}^{\prime}\right) \cdot\left(\mathbf{x}-\mathbf{x}^{\prime}\right)\right] \\
& \times \exp \left[-\frac{\sigma^{2}}{8}\left|\mathbf{k}-\mathbf{k}^{\prime}\right|^{2}\right] \\
& \times \exp \left[-\frac{1}{2 \sigma^{2}}\left|\mathbf{x}-\mathbf{x}^{\prime}\right|^{2}\right],
\end{aligned}
$$

where in calculating this expression we have extended the limits of integration in (2.12) to infinity. Equation (2.14) shows clearly that as wave packets of this prescribed form become more separated within a given phase space, they also appear to be more orthogonal. We can also use (2.13) to calculate the approximate spatial dependence for such a Gaussian wave packet. Using (2.9) we find the following:

$$
\begin{aligned}
\psi_{m}(\mathbf{r}, \mathbf{x}, \mathbf{k}) \equiv & \langle\mathbf{r} \mid \psi(m, \mathbf{x}, \mathbf{k})\rangle \\
= & {\left[\frac{2}{\pi \sigma^{2}}\right]^{3 / 4} \exp \left[-\frac{1}{\sigma^{2}}|\mathbf{r}-\mathbf{x}|^{2}-i \mathbf{k} \cdot \mathbf{x}\right] } \\
& \times \varphi_{m}(\mathbf{k}, \mathbf{r})
\end{aligned}
$$

where we have assumed that the lattice periodic part of the Bloch state varies slowly with respect to $\mathbf{k}$. This expression shows that the Gaussian wave packet exhibits a Bloch-state spatial dependence weighted by a Gaussian envelope function. This obvious result is in direct analogy to the minimum-uncertainty wave-packet states which can be easily derived for a free particle. ${ }^{15}$ The reader is also referred to a discussion of minimum-uncertainty packets by Stoler. ${ }^{16}$
We now return to a discussion of wave-packet states in general and define field operators associated with these states. These operators are given by

$$
\begin{aligned}
& \psi(m, \mathbf{x}, \mathbf{k}, t) \equiv \int d \mathbf{j} g^{*}(\mathbf{j}, \mathbf{k}) e^{i \mathbf{j} \cdot \mathbf{x}} a_{m}(\mathbf{j}, t), \\
& \psi^{\dagger}(m, \mathbf{x}, \mathbf{k}, t) \equiv \int d \mathbf{j} g(\mathbf{j}, \mathbf{k}) e^{-i \mathbf{j} \cdot \mathbf{x}} a_{m}^{\dagger}(\mathbf{j}, t)
\end{aligned}
$$

with associated anticommutation relations

$$
\left\{\psi(m, \mathbf{x}, \mathbf{k}, t), \psi^{\dagger}\left(n, \mathbf{x}^{\prime}, \mathbf{k}^{\prime}, t\right)\right\}=\delta_{m n}\left\langle\psi(m, \mathbf{x}, \mathbf{k}) \mid \psi\left(m, \mathbf{x}^{\prime}, \mathbf{k}^{\prime}\right)\right\rangle,
$$

$\left\{\psi(m, \mathbf{x}, \mathbf{k}, t), \psi\left(n, \mathbf{x}^{\prime}, \mathbf{k}^{\prime}, t\right)\right\}=0$,

$\left\{\psi^{\dagger}(m, \mathbf{x}, \mathbf{k}, t), \psi^{\dagger}\left(n, \mathbf{x}^{\prime}, \mathbf{k}^{\prime}, t\right)\right\}=0$

which are easily verified using (2.2), (2.3), and (2.4); and (2.12), (2.16), and (2.17). $\psi^{\dagger}(m, \mathbf{x}, \mathbf{k}, t)$ and $\psi(m, \mathbf{x}, \mathbf{k}, t)$ can be interpreted as operators which create and destroy a wave packet $|\psi(m, \mathbf{x}, \mathbf{k})\rangle$, a simple test of which is to apply $\psi^{\dagger}(m, \mathbf{x}, \mathbf{k}, t)$ to the vacuum state $|0\rangle$. In addition, by using (2.16) and (2.17), we can also show that

$$
\int d \mathbf{x} d \mathbf{k} \psi^{\dagger}(m, \mathbf{x}, \mathbf{k}, t) \psi(m, \mathbf{x}, \mathbf{k}, t)=\int d \mathbf{j} a_{m}^{\dagger}(\mathbf{j}, t) a_{m}(\mathbf{j}, t)
$$

from which $\psi^{\dagger}(m, \mathbf{x}, \mathbf{k}, t) \psi(m, \mathbf{x}, \mathbf{k}, t)$ is interpreted as an operator giving the number density of electrons at a point $(\mathbf{x}, \mathbf{k})_{m}$ in the phase space associated with band $m$.

As is the case with $a_{m}^{\dagger}(\mathbf{j}, t)$ and $a_{m}(\mathbf{j}, t)$, any operator can be expressed in terms of $\psi^{\dagger}(m, \mathbf{x}, \mathbf{k}, t)$ and $\psi(m, \mathbf{x}, \mathbf{k}, t)$. Consider, for example, a one-body Hamiltonian. Using completeness relation (2.10) twice we find

$$
\begin{gathered}
H=\sum_{m, n} \int d \mathbf{x} d \mathbf{x}^{\prime} d \mathbf{k} d \mathbf{k}^{\prime} M_{m n}\left(\mathbf{x}, \mathbf{x}^{\prime}, \mathbf{k}, \mathbf{k}^{\prime}\right) \\
\times \psi^{\dagger}(m, \mathbf{x}, \mathbf{k}, t) \psi\left(n, \mathbf{x}^{\prime}, \mathbf{k}^{\prime}, t\right)
\end{gathered}
$$

where a matrix element connecting points $(\mathbf{x}, \mathbf{k})_{m}$ and $\left(\mathbf{x}^{\prime}, \mathbf{k}^{\prime}\right)_{n}$ in phase spaces $m$ and $n$ has been defined as

$$
M_{m n}\left(\mathbf{x}, \mathbf{x}^{\prime}, \mathbf{k}, \mathbf{k}^{\prime}\right) \equiv\left\langle\psi(m, \mathbf{x}, \mathbf{k})|H| \psi\left(n, \mathbf{x}^{\prime}, \mathbf{k}^{\prime}\right)\right\rangle .
$$

To conclude the definitions associated with the wavepacket states we give the inverted forms of (2.9), (2.16), and (2.17). They are

$$
\begin{aligned}
|m \mathbf{j}\rangle & =\int d \mathbf{x} d \mathbf{k} g^{*}(\mathbf{j}, \mathbf{k}) e^{i \mathbf{j} \cdot \mathbf{x}}|\psi(m, \mathbf{x}, \mathbf{k})\rangle \\
a_{m}^{\dagger}(\mathbf{j}, t) & =\int d \mathbf{x} d \mathbf{k} g^{*}(\mathbf{j}, \mathbf{k}) e^{i \mathbf{j} \cdot \mathbf{x}} \psi^{\dagger}(m, \mathbf{x}, \mathbf{k}, t) \\
a_{m}(\mathbf{j}, t) & =\int d \mathbf{x} d \mathbf{k} g(\mathbf{j}, \mathbf{k}) e^{-i \mathbf{j} \cdot \mathbf{x}} \psi(m, \mathbf{x}, \mathbf{k}, t)
\end{aligned}
$$

In contrast to the electronic system, each lasing mode will be treated using the standard delocalized annihilation and creation operators which result from quantizing a noninteracting optical mode (for an alternate approach related to the wave-packet formalism of this treatment, see Ref. 17 which contains an interesting application of optical wave-packet operators). Proceeding in the normal fashion we quantize the vector potential as

$$
\mathbf{A}(\mathbf{r}, t)=\sum_{l}\left[\frac{\hbar}{2 \epsilon \omega_{l}}\right]^{1 / 2}\left[b_{l}(t)+b_{l}^{\dagger}(t)\right] \mathbf{u}_{l}(\mathbf{r})
$$


where

$$
\begin{aligned}
& \int d \mathbf{r} \mathbf{u}_{l}(\mathbf{r}) \cdot \mathbf{u}_{m}(\mathbf{r})=\delta_{l m}, \\
& {\left[b_{l}(t), b_{m}^{\dagger}(t)\right]=\delta_{l m},} \\
& {\left[b_{l}(t), b_{m}(t)\right]=0,} \\
& {\left[b_{l}^{\dagger}(t), b_{m}^{\dagger}(t)\right]=0,}
\end{aligned}
$$

and where [, ] signifies commutation. This simplified approach neglects any spatial modification of the mode which results from its interaction with the gain medium. For facet reflectivities greater than $30 \%$, however, these modifications are not severe. ${ }^{18}$

The model envisioned throughout this analysis is typical of state-of-the-art double heterostructure SL's. In it, an active layer, having optical and carrier confining qualities, has properties which are described in terms of the electronic wave-packet operators discussed above. The wave packets interact with the optical modes via dipole transitions and also with bulk crystal wave packets, phonons, crystal defects, and among themselves by various scattering mechanisms which produce the observed transport phenomena. The lasing modes, besides interacting with the electronic wave packets in the crystal, also interact with the free radiation modes since the resonator has a finite $Q$. Even though nearly all of these aspects of the model will eventually be accounted for in this treatment, the "system" in our model consists only of the active layer wave packets and the lasing optical modes. All other interactions will be referred to as bath interactions; and damping and fluctuations which result from these interactions will be included by using the quantummechanical fluctuation-dissipation theorem which is discussed in Sec. III. As such, the system Hamiltonian accounts only for the noninteracting electronic wave packets, the lasing modes, and the mutual interaction between these subsystems. Using the one-body representation (2.22), this Hamiltonian is given by

$$
\begin{gathered}
H=\sum_{m, n} \int d \mathbf{x} d \mathbf{x}^{\prime} d \mathbf{k} d \mathbf{k}^{\prime} M_{m n}^{e}\left(\mathbf{x}, \mathbf{x}^{\prime}, \mathbf{k}, \mathbf{k}^{\prime}\right) \\
\times \psi^{\dagger}(m, \mathbf{x}, \mathbf{k}, t) \psi\left(n, \mathbf{x}^{\prime}, \mathbf{k}^{\prime}, t\right) \\
+\sum_{m, n} \int d \mathbf{x} d \mathbf{x}^{\prime} d \mathbf{k} d \mathbf{k}^{\prime} M_{m n}^{I}\left(\mathbf{x}, \mathbf{x}^{\prime}, \mathbf{k}, \mathbf{k}^{\prime}\right) \\
\times \psi^{\dagger}(m, \mathbf{x}, \mathbf{k}, t) \psi\left(n, \mathbf{x}^{\prime}, \mathbf{k}^{\prime}, t\right) \\
+\sum_{l} \hbar \omega_{l}\left[b_{l}^{\dagger}(t) b_{l}(t)+\frac{1}{2}\right]
\end{gathered}
$$

where $\boldsymbol{M}_{m n}^{e}$ is the free-electron part,

$$
\boldsymbol{M}_{m n}^{e}\left(\mathbf{x}, \mathbf{x}^{\prime}, \mathbf{k}, \mathbf{k}^{\prime}\right) \equiv\left\langle\psi(m, \mathbf{x}, \mathbf{k})|(T+V)| \psi\left(n, \mathbf{x}^{\prime}, \mathbf{k}^{\prime}\right)\right\rangle,
$$

and $M_{m n}^{I}$ is the electron-radiation interaction part,

$$
M_{m n}^{I}\left(\mathbf{x}, \mathbf{x}^{\prime}, \mathbf{k}, \mathbf{k}^{\prime}\right) \equiv\left\langle\psi(m, \mathbf{x}, \mathbf{k})\left|-\frac{e}{m} \mathbf{A}(\mathbf{x}) \cdot \mathbf{p}\right| \psi\left(n, \mathbf{x}^{\prime}, \mathbf{k}^{\prime}\right)\right\rangle .
$$

These matrix elements give transition amplitudes between points $(\mathbf{x}, \mathbf{k})_{m}$ and $\left(\mathbf{x}^{\prime}, \mathbf{k}^{\prime}\right)_{n}$ in the semiclassical phase spaces. In their present form, however, they appear to only complicate matters over a treatment based on Bloch states. To benefit from this formalism two approximations must be made. Both take advantage of the localization in $\mathbf{x}$ and $\mathbf{k}$ of the electronic wave-packet states. The first is an approximation of the electronic matrix elements. For $M_{m n}^{e}$ we write

$$
M_{m n}^{e}\left(\mathbf{x}, \mathbf{x}^{\prime}, \mathbf{k}, \mathbf{k}^{\prime}\right) \approx \epsilon_{m}(\mathbf{k})\left\langle\psi(m, \mathbf{x}, \mathbf{k}) \mid \psi\left(m, \mathbf{x}^{\prime}, \mathbf{k}^{\prime}\right)\right\rangle \delta_{m n},
$$

that is, we assume the wave-packet states are approximately electronic eigenstates of the unperturbed crystal. Such an assumption is justified provided the wave-packet states span only a small volume in crystal momentum space. For $M_{m n}^{I}$ we write

$$
\begin{aligned}
& M_{m n}^{I}\left(\mathbf{x}, \mathbf{x}^{\prime}, \mathbf{k}, \mathbf{k}^{\prime}, t\right) \\
& \approx-\frac{e}{m} \sum_{l}\left[\frac{\hbar}{2 \epsilon \omega_{l}}\right]^{1 / 2}\left(b_{l}+b_{l}^{\dagger}\right) \\
& \quad \times \mathbf{u}_{l}(\mathbf{x}) \cdot\left\langle\psi(m, \mathbf{x}, \mathbf{k})|\mathbf{p}| \psi\left(n, \mathbf{x}^{\prime}, \mathbf{k}^{\prime}\right)\right\rangle \\
& \approx \sum_{l} h_{m n}(\mathbf{k}) u_{l}(\mathbf{x})\left(b_{l}+b_{l}^{\dagger}\right)\left\langle\psi(m, \mathbf{x}, \mathbf{k}) \mid \psi\left(m, \mathbf{x}^{\prime}, \mathbf{k}^{\prime}\right)\right\rangle,
\end{aligned}
$$

where the vector nature of $\mathbf{u}_{l}(\mathbf{x})$ has been absorbed into $h_{m n}(\mathbf{k})$. In (2.36), the slowly varying spatial dependence of the optical field in comparison to the electronic wave packets has been used to remove the optical space variation $\mathbf{u}(\mathbf{r})$ outside the wave-packet bras and kets-the same approximation which comprises the dipole approximation in an atomic system. In (2.36'), the well-defined momentum of the wave packets has been used again. Here we approximate a " $k$ "-conserving interband absorption or emission of a photon as a transition $(\mathbf{x}, \mathbf{k})_{m} \rightarrow(\mathbf{x}, \mathbf{k})_{n}$ between phase spaces $m$ and $n$ which conserves the semi-

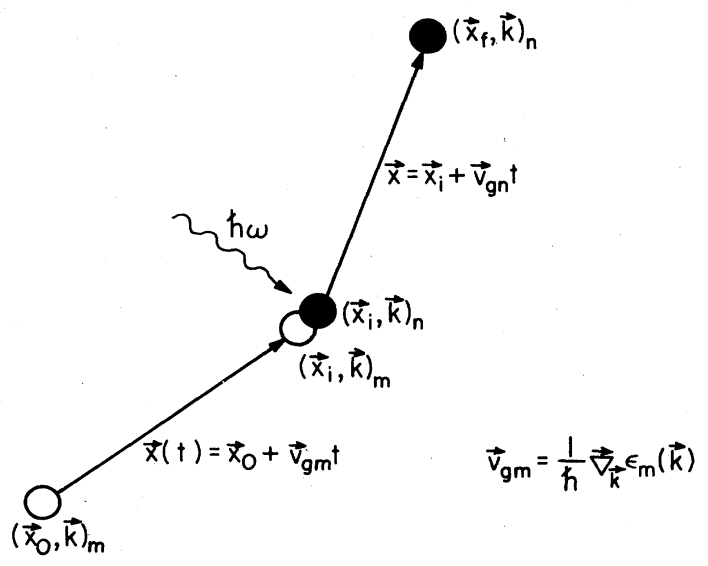

FIG. 2. Intuitive picture of photon absorption by a wave packet propagating in band $m$ with group velocity $\mathbf{v}_{g m}(\mathbf{k})$ resulting in creation of a wave packet in band $n$ having group velocity $\mathbf{v}_{g n}(\mathbf{k})$ (and annihilation of the wave packet in band $m$ ). 
classical crystal momentum and in addition leaves the electron position unchanged. That is, the wave packets are assumed to have enough "Bloch" character, by virtue of their microscopic largeness in $\mathrm{x}$ space, so that a $k$ conserving transition between Bloch states remains approximately a $k$-conserving transition between wavepacket states (see Fig. 2). To first order the quantities $\epsilon_{m}(\mathbf{k})$ and $h_{m n}(\mathbf{k})$ are, in fact, the quantities found using the true Bloch eigenstates; the discrepancy becoming smaller as the electronic wave packets encompass larger volumes of the crystal. On the other hand, this volume cannot become so large that optical mode features or transport features become imperceptible. Thus, the spatial breadth $\sigma$ of the electronic wave packets must satisfy

$$
a<<\sigma<\min \left(\lambda, \lambda_{D}\right),
$$

where $a$ is a typical lattice constant, $\lambda$ is the optical wavelength in the crystal, and $\lambda_{D}$ is the diffusion length. This, of course, is just the condition for wave-packet microscopic largeness and macroscopic smallness which was discussed earlier. Higher-order corrections to the matrix elements can be calculated by using (2.9) combined with Taylor-series expansion of the Hamiltonian about the center-of-mass coordinates. As an example, the first two terms in such an expansion of Eq. (2.33) are shown below

$$
\begin{aligned}
M_{m n}^{e}\left(\mathbf{x}, \mathbf{x}^{\prime}, \mathbf{k}, \mathbf{k}^{\prime}\right)= & \delta_{m n} \epsilon_{m}(\mathbf{k})\left\langle\psi(m, \mathbf{x}, \mathbf{k}) \mid \psi\left(m, \mathbf{x}^{\prime}, \mathbf{k}^{\prime}\right)\right\rangle \\
+ & \delta_{m n} e^{i \mathbf{k} \cdot \boldsymbol{\xi}}\left[\nabla_{\mathbf{k}} \epsilon_{m}(\mathbf{k}) \cdot \nabla_{\xi}\right] e^{-i \mathbf{k} \cdot \boldsymbol{\xi}} \\
& \times\left\langle\psi(m, \mathbf{x}, \mathbf{k}) \mid \psi\left(m, \mathbf{x}^{\prime}, \mathbf{k}^{\prime}\right)\right\rangle
\end{aligned}
$$

where $\boldsymbol{\xi} \equiv \mathbf{x}-\mathbf{x}^{\prime}$.

Provided condition (2.37) holds, the wave-packet states appear highly localized in the various phase spaces in comparison to variations of the Hamiltonian. This also implies that the dynamic variables will vary slowly, allowing the following additional approximation to be made:

$$
\left\langle\psi(m, \mathbf{x}, \mathbf{k}) \mid \psi\left(n, \mathbf{x}^{\prime}, \mathbf{k}^{\prime}\right)\right\rangle \rightarrow M \delta_{m n} D\left(\mathbf{x}-\mathbf{x}^{\prime}\right) D\left(\mathbf{k}-\mathbf{k}^{\prime}\right) .
$$

Basically, this is a multipole expansion of $\left\langle\psi(m, \mathbf{x}, \mathbf{k}) \mid \psi\left(n, \mathbf{x}^{\prime}, \mathbf{k}^{\prime}\right)\right\rangle$ in which only the first term is retained. The coefficient $M$ is the monopole term in this expansion and will depend on the specific form chosen for the wave-packet states. For simplicity, we take $M=1$ in this analysis. In retaining only the first term in the multipole expansion, we are in a sense taking the analysis to the semiclassical picture of the Bloch electron. Unlike the semiclassical picture, however, we now have a means of including interband transitions and other quantum transitions satisfying condition (2.37) in a quantummechanically rigorous fashion. In addition, the Pauli exclusion principle is maintained through the anticommutation relations,

$$
\left\{\psi(m, \mathbf{x}, \mathbf{k}, t), \psi^{\dagger}\left(n, \mathbf{x}^{\prime}, \mathbf{k}^{\prime}, t\right)\right\}=\delta_{m n} D\left(\mathbf{x}-\mathbf{x}^{\prime}\right) D\left(\mathbf{k}-\mathbf{k}^{\prime}\right),
$$

$$
\left\{\psi(m, \mathbf{x}, \mathbf{k}, t), \psi\left(n, \mathbf{x}^{\prime}, \mathbf{k}^{\prime}, t\right)\right\}=0
$$

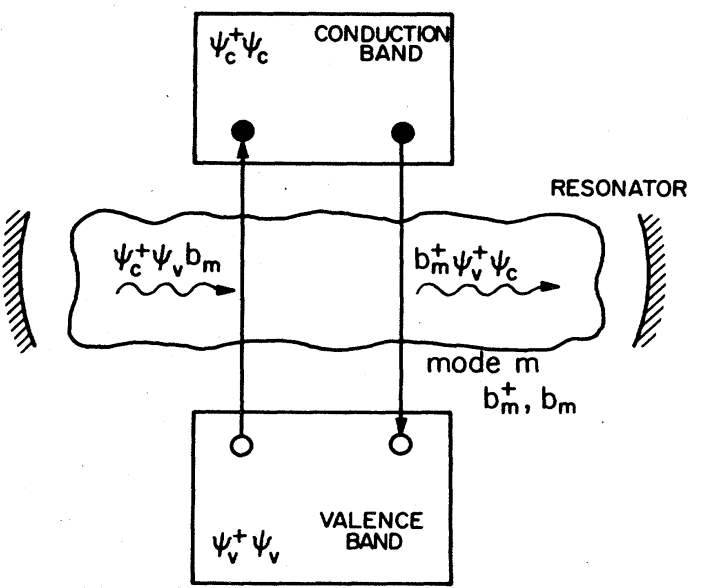

FIG. 3. System model for a semiconductor laser showing components and dynamic variables. Bath interactions are not included in the diagram.

$$
\left\{\psi^{\dagger}(m, \mathbf{x}, \mathbf{k}, t), \psi^{\dagger}\left(n, \mathbf{x}^{\prime}, \mathbf{k}^{\prime}, t\right)\right\}=0,
$$

where the first relation is an approximation of (2.18) using (2.39), but the latter two relations remain exact.

Using (2.35) and (2.36) in (2.32), and then simplifying the result using (2.39) gives for the system Hamiltonian,

$$
\begin{gathered}
H=\sum_{m} \int d \mathbf{x} d \mathbf{k} \epsilon_{m}(\mathbf{k}) \psi^{\dagger}(m, \mathbf{x}, \mathbf{k}, t) \psi(m, \mathbf{x}, \mathbf{k}, t) \\
+\sum_{l} \hbar \omega_{l}\left[b_{l}^{\dagger}(t) b_{l}(t)+\frac{1}{2}\right] \\
+\sum_{l, m, n} \int d \mathbf{x} d \mathbf{k} h_{m n}(\mathbf{k}) u_{l}(\mathbf{x})\left[b_{l}(t)+b_{l}^{\dagger}(t)\right] \\
\quad \times \psi^{\dagger}(m, \mathbf{x}, \mathbf{k}, t) \psi(n, \mathbf{x}, \mathbf{k}, t) .
\end{gathered}
$$

We specialize this Hamiltonian to a two-band model (see Fig. 3),

$$
\begin{aligned}
H= & \int d \mathbf{x} d \mathbf{k}\left[\epsilon_{c}(\mathbf{k}) \psi_{c}^{\dagger} \psi_{c}+\epsilon_{v}(\mathbf{k}) \psi_{v}^{\dagger} \psi_{v}\right] \\
& +\sum_{l} \hbar \omega_{l}\left(b_{l}^{\dagger} b_{l}+\frac{1}{2}\right) \\
& +\sum_{l} \int d \mathbf{x} d \mathbf{k} u_{l}(\mathbf{x})\left(b_{l}+b_{l}^{\dagger}\right)\left[h(\mathbf{k}) \psi_{c}^{\dagger} \psi_{v}+h^{*}(\mathbf{k}) \psi_{v}^{\dagger} \psi_{c}\right]
\end{aligned}
$$

where coordinate and temporal dependences have been suppressed in the dynamic variables.

\section{EQUATIONS OF MOTION}

The system Hamiltonian (2.44) includes the unperturbed energy terms of the Bloch electrons and the lasing modes as well as their interaction energy. The time evolution predicted by this Hamiltonian represents a zerothorder description of the laser, which although able to account for stimulated emission and absorption is seriously deficient in describing areas such as pumping and cavity loss. The missing terms in the system Hamiltonian responsible for these effects and others come under the heading of bath interactions: additional energy terms 
stemming from scattering of the system with other systems having a large number of degrees of freedom. The complete time-evolution equation for an operator $A$ could thus be envisioned as

$$
\frac{d A}{d t}=\frac{i}{\hbar}\left[H_{0}+H_{I}+H_{B}, A\right],
$$

where $H_{0}$ and $H_{I}$ are the unperturbed energy and interaction energy terms in the system Hamiltonian (2.44) and $H_{B}$ comprises the various bath interactions. In this section we will develop a set of operator equations of motion governing lasing action and transport phenomena in SL's. We incorporate bath interactions into these equations in a standard way which is described below.

The concepts of system and bath are of profound importance in thermodynamics and statistical mechanics. The system lies at the focus of attention; its thermodynamic state variables or dynamic variables, as the case may be, are to be measured or calculated. The bath, on the other hand, has consequence only through changes it causes in the system by interacting with it. It is normally assumed to have a well-defined thermodynamic state, which owing to its enormous size relative to the system, is unaffected by interaction with the system. These definitions put no restriction on the system so long as the bath or baths it interacts with can be chosen to be much larger than the system. In the present case, however, we assume the label "system" to imply, in addition to the above properties, an analytically manageable set of dynamic variables; specifically, the operators $\psi_{c}^{\dagger} \psi_{c}, \psi_{v}^{\dagger} \psi_{v}, \psi_{v}^{\dagger} \psi_{c},\left\{b_{l}^{\dagger}\right\}$, and $\left\{b_{l}\right\}$ which under the system Hamiltonian (2.44) constitute a self-consistent set. These operators interact with three independent baths: the conduction band, valence band, and free radiation baths. For the moment we consider the effect of these bath interactions in the absence of interactions between the components of the system [i.e., setting $H_{I}=0$ in (3.1)]. Without any interactions (i.e., setting $H_{I}=0$ and $H_{B}=0$ ), the system equations of motion are

$$
\begin{aligned}
& \dot{b}_{l}^{\dagger}=i \omega_{l} b_{l}^{\dagger}, \\
& \frac{d \psi_{c}^{\dagger} \psi_{v}}{d t}=i \Omega(\mathbf{k}) \psi_{c}^{\dagger} \psi_{v}, \\
& \frac{d \psi_{c}^{\dagger} \psi_{c}}{d t}=0, \\
& \frac{d \psi_{v}^{\dagger} \psi_{v}}{d t}=0,
\end{aligned}
$$

where

$$
\Omega(\mathbf{k}) \equiv \frac{\epsilon_{c}(\mathbf{k})-\epsilon_{v}(\mathbf{k})}{\hbar}
$$

and where equations for $b_{l}$ and $\psi_{v}^{\dagger} \psi_{c}$ are merely the Hermitian adjoints of (3.2) and (3.3). Bath terms in the Hamiltonian affect these equations first by driving the equilibration or damping of the dynamic variables and second by superimposing fluctuations on their damped motion. Damping results from the enormous number of degrees of freedom in the various baths, making it highly unlikely that energy leaking out of the system will return from the baths. Fluctuations, on the other hand, are the result of bath energy which "couples into" or "pumps" the system. The basic problem is to incorporate these two aspects of the system-bath interaction into the equations of motion. One approach to this problem is to consider the systembath interaction explicitly. It is clear, however, that in most cases such an approach is prohibitively complicated. Another approach, the one employed here, is to add damping phenomenologically to the system equations of motion and then to determine fluctuations with the quantum-mechanical fluctuation-dissipation theorem. We give only a basic outline of this theorem below, deferring a more rigorous explanation to comprehensive treatments of the subject by $\mathrm{Lax}^{19}$ and Haken et al. ${ }^{20}$ Our particular formulation follows Lax's treatment.

The fluctuation-dissipation theorem has both classical and quantum forms. In both cases it accounts for fluctuations caused by thermal energy coupling into a chosen system from a bath. In the quantum case, however, it is also essential to preserving the quantum-mechanical nature of the damped system by maintaining the canonical commutation relations of the dynamic variables. The theorem presumes that damping can be incorporated in some way into the dynamic equations (normally phenomenologically). The mean (i.e. quantum average over all baths) motion of the dynamic variables $\left\{A_{i}\right\}$ is then given by

$$
\frac{d\left\langle A_{i}\right\rangle}{d t}=\left\langle\Gamma_{i}\left(\left\{A_{j}\right\}\right)\right\rangle,
$$

where $\left\{\Gamma_{i}\right\}$ are a set of transport functions which include damping. The unaveraged form of (3.7) contains a fluctuation operator to account for the thermal nature of the bath energy which couples into the system. Thus, the unaveraged form of (3.7) appears as follows:

$$
\frac{d A_{i}}{d t}=\Gamma_{i}\left(\left\{A_{j}\right\}\right)+f_{i}(t),
$$

where, on account of (3.7), the fluctuation operator $f_{i}(t)$ must vanish upon bath averaging,

$$
\left\langle f_{i}(t)\right\rangle=0 \text {. }
$$

The stochastic operators $\left\{f_{i}(t)\right\}$ are normally assumed to be Gaussian, resulting from a central limit argument, and in addition Markovian,

$$
\left\langle f_{i}(t+\tau) f_{i}(t)\right\rangle=W_{i j} D(\tau),
$$

where the normalization coefficient $W_{i j}$ is often referred to as a generalized diffusion constant since it does, in fact, so function in Einstein's model of Brownian motion. (Note: in this analysis we will assume the fluctuation operators are delta-correlated in phase space as well as in time.) The final and most important part of the fluctuation-dissipation theorem is that this diffusion constant can be determined from knowledge of the transport function in (3.7) by employing the generalized Einstein relation,

$$
W_{i j}=\frac{d\left\langle A_{i} A_{j}\right\rangle}{d t}-\left\langle\Gamma_{i}\left(\left\{A_{k}\right\}\right) A_{j}\right\rangle-\left\langle A_{i} \Gamma_{j}\left(\left\{A_{k}\right\}\right)\right\rangle,
$$


which can be derived quite readily from (3.8) and (3.10).

To recapitulate this approach consider the problem of incorporating damping and fluctuations into the opticalfield equation (see also Ref. 2). Reasonable selections for damped unpumped optical-field equations are

$$
\begin{aligned}
& \dot{b}_{l}^{\dagger}=\left(i \omega_{l}-\frac{1}{2 \tau_{l}}\right) b_{l}^{\dagger}+g_{l}^{\dagger}(t), \\
& \dot{b}_{l}=\left(-i \omega_{l}-\frac{1}{2 \tau_{l}}\right) b_{l}+g_{l}(t),
\end{aligned}
$$

where $\tau_{l}$ is the photon lifetime of lasing mode $l$ in the unpumped cavity, and $g_{l}^{\dagger}$ and $g_{l}$ are the fluctuation operators. To normalize these operators, the transport terms in (3.12) and (3.13) are used in the generalized Einstein relation. In steady state the results are

$$
\begin{aligned}
& \left\langle g_{l}(t+\tau) g_{m}(t)\right\rangle=0 \\
& \left\langle g_{l}^{\dagger}(t+\tau) g_{m}^{\dagger}(t)\right\rangle=0 \\
& \left\langle g_{l}^{\dagger}(t+\tau) g_{m}(t)\right\rangle=\frac{1}{\tau_{l}}\left\langle b_{l}^{\dagger} b_{m}\right\rangle D(\tau)=\frac{n_{l}}{\tau_{l}} D(\tau) \delta_{l m}, \\
& \left\langle g_{l}(t+\tau) g_{m}^{\dagger}(t)\right\rangle=\frac{1}{\tau_{l}}\left\langle b_{l} b_{m}^{\dagger}\right\rangle D(\tau)=\frac{n_{l}+1}{\tau_{l}} D(\tau) \delta_{l m},
\end{aligned}
$$

where $n_{l} \equiv\left\langle b_{l}^{\dagger} b_{l}\right\rangle$ is the number of thermal photons in the optical mode $l$ as given by

$$
n_{l}=\frac{1}{e^{\hbar \omega_{l} / k_{b} T_{R}}-1} .
$$

$T_{R}$ here is the temperature of the free radiation bath. It should be noted that lack of commutability between $b_{l}^{\dagger}$ and $b_{l}$ is reflected in the Einstein relations (3.16) and (3.17). This fact is central to preserving the quantum nature of $b_{l}^{\dagger}$ and $b_{l}$ in the damped system.

Both damping and fluctuations are introduced into Eqs. (3.3), (3.4), and (3.5) as follows:

$$
\begin{aligned}
& \frac{d \psi_{c}^{\dagger} \psi_{v}}{d t}=[i \Omega(\mathbf{k})-\gamma(\mathbf{x}, \mathbf{k})] \psi_{c}^{\dagger} \psi_{v}+\Delta(\mathbf{x}, \mathbf{k}, t) \\
& \frac{d \psi_{c}^{\dagger} \psi_{c}}{d t}=-\frac{\psi_{c}^{\dagger} \psi_{c}-f_{c}}{\tau_{c}(\mathbf{x}, \mathbf{k})}+L_{c}(\mathbf{x}, \mathbf{k}, t) \\
& \frac{d \psi_{v}^{\dagger} \psi_{v}}{d t}=-\frac{\psi_{v}^{\dagger} \psi_{v}-f_{v}}{\tau_{v}(\mathbf{x}, \mathbf{k})}+L_{v}(\mathbf{x}, \mathbf{k}, t)
\end{aligned}
$$

Each of these equations is driven by a Langevin fluctuation operator which is in general a function of time and location in the appropriate phase space. The second moments of these Langevin operators, found using (3.11), appear below:

$$
\begin{aligned}
& \left\langle\Delta^{\dagger}(\mathbf{x}, \mathbf{k}, t+\tau) \Delta^{\dagger}\left(\mathbf{x}^{\prime}, \mathbf{k}^{\prime}, t\right)\right\rangle=0, \\
& \left\langle\Delta^{\dagger}(\mathbf{x}, \mathbf{k}, t+\tau) \Delta\left(\mathbf{x}^{\prime}, \mathbf{k}^{\prime}, t\right)\right\rangle \\
& \quad=2 \gamma f_{v}\left[1-(2 \pi)^{3} f_{c}\right] D\left(\mathbf{x}-\mathbf{x}^{\prime}, \mathbf{k}-\mathbf{k}^{\prime}, \tau\right),
\end{aligned}
$$

$$
\begin{aligned}
& \begin{aligned}
\langle\Delta(\mathbf{x}, \mathbf{k}, t & \left.+\tau) \Delta^{\dagger}\left(\mathbf{x}^{\prime}, \mathbf{k}^{\prime}, t\right)\right\rangle \\
& =2 \gamma f_{c}\left[1-(2 \pi)^{3} f_{v}\right] D\left(\mathbf{x}-\mathbf{x}^{\prime}, \mathbf{k}-\mathbf{k}^{\prime}, \tau\right), \\
\left\langle L_{c}(\mathbf{x}, \mathbf{k}, t\right. & \left.+\tau) L_{c}\left(\mathbf{x}^{\prime}, \mathbf{k}^{\prime}, t\right)\right\rangle \\
& =\frac{2}{\tau_{c}} f_{c}\left[1-(2 \pi)^{3} f_{c}\right] D\left(\mathbf{x}-\mathbf{x}^{\prime}, \mathbf{k}-\mathbf{k}^{\prime}, \tau\right),
\end{aligned} \\
& \left\langle L_{v}(\mathbf{x}, \mathbf{k}, t+\tau) L_{v}\left(\mathbf{x}^{\prime}, \mathbf{k}^{\prime}, t\right)\right\rangle \\
& \quad=\frac{2}{\tau_{v}} f_{v}\left[1-(2 \pi)^{3} f_{v}\right] D\left(\mathbf{x}-\mathbf{x}^{\prime}, \mathbf{k}-\mathbf{k}^{\prime}, \tau\right), \\
& \left\langle L_{c}(\mathbf{x}, \mathbf{k}, t+\tau) L_{v}\left(\mathbf{x}^{\prime}, \mathbf{k}^{\prime}, t\right)\right\rangle=0 .
\end{aligned}
$$

Damping parameters also, in general, have a phase-space dependence caused by energy-dependent scattering rates and spatial inhomogeneities, such as a nonuniform carrier density. The damping parameter $\gamma(\mathbf{x}, \mathbf{k})$ describes collisionally induced loss of polarization between states in a transition. Between Bloch states in the conduction and valence bands the time $\gamma^{-1}$ is thought to be roughly 0.1 psec. $^{7}$

The damping form assumed in (3.20) and (3.21) is characteristic of the relaxation time approximation. ${ }^{8}$ These terms give the relaxation rate of $\psi_{c}^{\dagger} \psi_{c}$ and $\psi_{v}^{\dagger} \psi_{v}$ to their local quasiequilibrium forms given by $f_{c}$ and $f_{v}$ where, for example,

$$
\left\langle f_{c}\right\rangle \equiv \frac{1}{(2 \pi)^{3}} \frac{1}{\exp \left\{\left[\epsilon_{c}(\mathbf{k})-\eta_{c}(\mathbf{x}, t)\right] / k_{b} T(\mathbf{x}, t)\right\}+1}
$$

is the quasi-Fermi-distribution function for the conduction band with associated temporally and spatially varying temperature and electrochemical potential (to allow for the possibility of internal electric potentials). The factor involving $(2 \pi)^{3}$ is a normalization which enters into this expression since $f_{c}$ and $f_{v}$ as well as $\psi_{c}^{\dagger} \psi_{c}$ and $\psi_{v}^{\dagger} \psi_{v}$ represent occupancy densities in $(\mathbf{x}, \mathbf{k})$ phase spaces. In SL's local quasiequilibrium is established extremely rapidly by intraband scattering. The phenomenological relaxation times for this thermalization within each band are the $\tau_{c}(\mathbf{x}, \mathbf{k})$ and $\tau_{v}(\mathbf{x}, \mathbf{k})$ appearing in (3.20) and (3.21). These times are generally thought to lie in the range $0.1-1.0$ psec. $^{21}$ To be strictly correct we should also include thermal generation and spontaneous recombination rate terms in (3.20) and (3.21). These terms are utterly negligible, however, in comparison to the intraband scattering terms and are not considered for the moment. In fact, the absence of noticeable spectral hole burning in SL's implies that intraband scattering rates also dominate stimulated rate terms resulting from the electron-radiation interaction. $^{7,22}$ This extremely rapid thermalization is the basis for the assumption, often made, that local quasiequilibrium holds during lasing action. We shall assume that this is the case in the remainder of this analysis. Local quasiequilibrium of the conduction-band and valence-band baths is always assumed to hold and with it well-defined local temperature and quasielectrochemical potentials. Spatial equilibrium is not assumed, however, as its characteristic equilibration time is comparable to relaxation times governing photon-inversion dynamics. 
Currents which result from spatial nonequilibrium (pump currents included) are assumed to be expressible as gradients of the temperature distribution and quasielectrochemical potentials.

The assumption of local quasiequilibrium means that Eqs. (3.20) and (3.21) are approximately correct even when the electron-radiation interaction is turned on. The short-term motion of $\psi_{c}^{\dagger} \psi_{c}$ and $\psi_{v}^{\dagger} \psi_{v}$ therefore consists only of thermal fluctuations about $f_{c}$ and $f_{v}$. The longterm motion, including the action of stimulated rate terms, is absorbed almost completely by the quasi-Fermioperators $f_{c}$ and $f_{v}$. This kind of approximate picture is very similar to that found in the Born-Oppenheimer approximation. The idea is illustrated in Fig. 4. Figure 4(a) depicts a relaxation oscillation of $\left\langle f_{c}\right\rangle$ or $\left\langle f_{v}\right\rangle$ after the inversion has been disturbed from its operating point; Fig. 4(b) shows $\left\langle\psi_{c}^{\dagger} \psi_{c}\right\rangle$ or $\left\langle\psi_{v}^{\dagger} \psi_{v}\right\rangle$ during the same oscillation, the added fuzziness resulting from intraband thermal fluctuations. The slowly varying time evolution of the quasi-Fermi-operators can be found through application of the following obvious identities to (3.20) and (3.21):

$$
\begin{aligned}
& \int d \mathbf{k} f_{c}=\int d \mathbf{k} \psi_{c}^{\dagger} \psi_{c}, \\
& \int d \mathbf{k} f_{v}=\int d \mathbf{k} \psi_{v}^{\dagger} \psi_{v} .
\end{aligned}
$$

A further simplification can be made by assuming that intraband scattering preserves the carrier density (there will, however, be fluctuations about an average value stemming from shot contributions of the balanced intraband scattering rates into and out of a particular location),

$$
\begin{gathered}
\int d \mathbf{k} \frac{\psi_{c}^{\dagger} \psi_{c}-f_{c}}{\tau_{c}(\mathbf{x}, \mathbf{k})}=0 \\
\int d \mathbf{k} \frac{\psi_{v}^{\dagger} \psi_{v}-f_{v}}{\tau_{v}(\mathbf{x}, \mathbf{k})}=0
\end{gathered}
$$

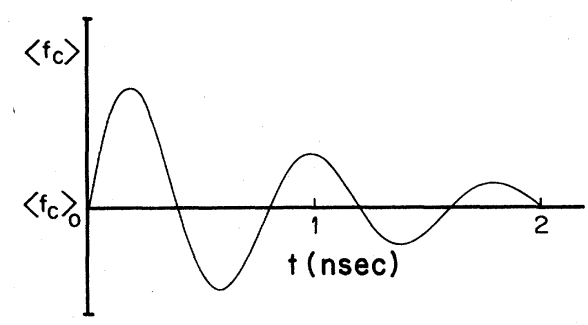

(a)

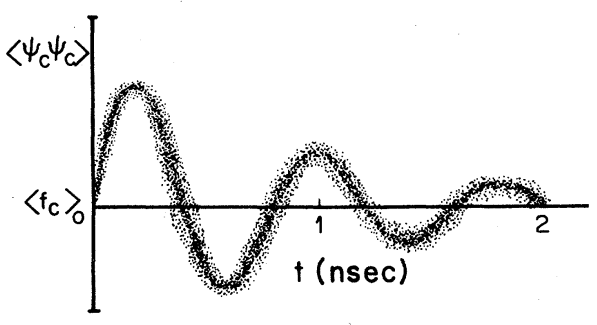

(b)

FIG. 4. A relaxation oscillation of the Fermi number density $\left\langle f_{c}\right\rangle$ and the phase-space number density $\left\langle\psi_{c}^{\dagger} \psi_{c}\right\rangle$ towards the operating point value $\left\langle f_{c}\right\rangle_{0}$. Intraband scattering produces the added fuzziness in the lower plot.
Equations (3.12), (3.19), (3.20), and (3.21) describe the motion of the chosen dynamic variables under the Hamiltonian $H_{0}+H_{B}$. We now include the electron-radiation interaction term given in (2.44). Neglecting nonsynchronous terms, the resulting dynamic equations are

$$
\begin{aligned}
\dot{b}_{l}^{\dagger}= & {\left[i \omega_{l}-\frac{1}{2 \tau_{l}}\right] b_{l}^{\dagger} } \\
& +\frac{i}{\sqrt{2 \epsilon \hbar \omega_{l}}} \int d \mathbf{x} d \mathbf{k} u_{l}(\mathbf{x}) q(\mathbf{k}) \psi_{c}^{\dagger} \psi_{v}+g_{l}^{\dagger}(t) \\
\frac{d \psi_{c}^{\dagger} \psi_{v}}{d t}= & {[i \Omega(\mathbf{k})-\gamma(\mathbf{x}, \mathbf{k})] \psi_{c}^{\dagger} \psi_{v} } \\
& -i \sum_{l} \frac{u_{l}(\mathbf{x}) q^{*}(\mathbf{k})}{\sqrt{2 \epsilon \hbar \omega_{l}}}\left(\psi_{c}^{\dagger} \psi_{c}-\psi_{v}^{\dagger} \psi_{v}\right) b_{l}^{\dagger} \\
& +\Delta(\mathbf{x}, \mathbf{k}, t) \\
\frac{d \psi_{c}^{\dagger} \psi_{c}}{d t}= & -\frac{\psi_{c}^{\dagger} \psi_{c}-f_{c}}{\tau_{c}(\mathbf{x}, \mathbf{k})}+Y+L_{c}(\mathbf{x}, \mathbf{k}, t) \\
\frac{d \psi_{v}^{\dagger} \psi_{v}}{d t}= & -\frac{\psi_{v}^{\dagger} \psi_{v}-f_{v}}{\tau_{v}(\mathbf{x}, \mathbf{k})}-Y+L_{v}(\mathbf{x}, \mathbf{k}, t)
\end{aligned}
$$

where

$$
\begin{aligned}
q(\mathbf{k}) & \equiv \frac{h(\mathbf{k})}{\hbar}, \\
Y \equiv & -R(\mathbf{x}, \mathbf{k})+G(\mathbf{x}, \mathbf{k}) \\
& -i \sum_{l} \frac{u_{l}(\mathbf{x})}{\sqrt{2 \epsilon \hbar \omega_{l}}}\left[q(\mathbf{k}) \psi_{c}^{\dagger} \psi_{v} b_{l}-q^{*}(\mathbf{k}) b_{l}^{\dagger} \psi_{v}^{\dagger} \psi_{c}\right]
\end{aligned}
$$

$G(\mathbf{x}, \mathbf{k})$ and $R(\mathbf{x}, \mathbf{k})$ are generation and spontaneous recombination terms whose explicit dependence on system operators is not important for our purposes. From the above discussion, the operator $Y$ can be omitted in (3.35) and (3.36) for consideration of the rapid variations of $\psi_{c}^{\dagger} \psi_{c}$ and $\psi_{v}^{\dagger} \dot{\psi}_{v}$, whereas the slow variations of the quasiFermi-levels (or equivalently the carrier density) can be determined by application of (3.29) and (3.31) to (3.35),

$$
\frac{d n}{d t}=\int d \mathbf{k} Y+\int d \mathbf{k} L_{c}(\mathbf{x}, \mathbf{k}, t)
$$

where

$$
n(\mathbf{x}, t) \equiv \int d \mathbf{k} f_{c}=\int d \mathbf{k} \psi_{c}^{\dagger} \psi_{c}
$$

is the electron density operator. The corresponding equation for the valence band, found by applying (3.30) and (3.32) to (3.36), is omitted since it is determined from knowledge of $f_{c}$ [or $n(\mathbf{x}, t)$ ] with the quasineutrality condition. We now rewrite (3.39) using (3.38) and also reexpress the total time derivative in the form of a conservation equation 


$$
\begin{aligned}
\partial_{t} n+\nabla \cdot \mathbf{J}= & -R(\mathbf{x})+G(\mathbf{x}) \\
& -i \sum_{l} \frac{u_{l}(\mathbf{x})}{\sqrt{2 \epsilon \hbar \omega_{l}}} \int d \mathbf{k}\left[q(\mathbf{k}) \psi_{c}^{\dagger} \psi_{v} b_{l}\right. \\
& \left.-q^{*}(\mathbf{k}) b_{l}^{\dagger} \psi_{v}^{\dagger} \psi_{c}\right] \\
& +\int d \mathbf{k} L_{c}(\mathbf{x}, \mathbf{k}, t),
\end{aligned}
$$

where

$$
\begin{aligned}
& R(\mathbf{x}) \equiv \int d \mathbf{k} R(\mathbf{x}, \mathbf{k}), \\
& G(\mathbf{x}) \equiv \int d \mathbf{k} G(\mathbf{x}, \mathbf{k}),
\end{aligned}
$$

and where $\mathbf{J}$ is the electron current-density operator caused by drift and diffusion, for example. Equation (3.41) is a carrier-density operator rate equation which can be used to study both normal electronic transport in conjunction with electron-radiation induced interband transitions.

We assume it is possible to define drift and diffusion coefficients. A more fundamental approach would begin with the operator version of the Boltzmann transport equation given by Eq. (3.35),

$$
\begin{aligned}
\frac{d \psi_{c}^{\dagger} \psi_{c}}{d t} & =\partial_{t} \psi_{c}^{\dagger} \psi_{c}+\mathbf{v}_{g} \cdot \nabla_{\mathbf{x}} \psi_{c}^{\dagger} \psi_{c}+\mathbf{k} \cdot \nabla_{\mathbf{k}} \psi_{c}^{\dagger} \psi_{c} \\
& =-\frac{\psi_{c}^{\dagger} \psi_{c}-f_{c}}{\tau_{c}}+Y+L_{c},
\end{aligned}
$$

where $\mathbf{v}_{\mathbf{g}}=\mathbf{x}$ is the group velocity of a wave packet. Using this equation one could derive formulas for the transport coefficients. For a related discussion see Ref. 8; also see Refs. 11-13 in regard to operator transport equations.

\section{THE RATE-EQUATION APPROXIMATION}

Normally, it is possible to make one simplification of Eqs. (3.33)-(3.36) and (3.41) without much loss of generality. To do this Eq. (3.34) is solved for the operator $\psi_{c}^{\dagger} \psi_{v}$ by employing the rate-equation approximation. Integrating the total time derivative in (3.34) yields

$$
\begin{aligned}
\psi_{c}^{\dagger} \psi_{v}= & -i \sum_{l} \int_{-\infty}^{t} d t^{\prime} \frac{u_{l}\left(\mathbf{x}^{\prime}\right) q^{*}\left(\mathbf{k}^{\prime}\right)}{\sqrt{2 \epsilon \hbar \omega_{l}}}\left(\psi_{c}^{\dagger} \psi_{c}-\psi_{v}^{\dagger} \psi_{v}\right) b_{l}^{\dagger} \\
& \times \exp \left\{\left[i \Omega\left(\mathbf{k}^{\prime}\right)-\gamma\right]\left(t-t^{\prime}\right)\right\} \\
& +\int_{-\infty}^{t} d t^{\prime} \Delta\left(\mathbf{x}^{\prime}, \mathbf{k}^{\prime}, t^{\prime}\right) \exp \left\{\left[i \Omega\left(\mathbf{k}^{\prime}\right)-\gamma\right]\left(t-t^{\prime}\right)\right\} .
\end{aligned}
$$

As mentioned in Sec. III the damping parameter $\gamma$ is of the order of $0.1 \mathrm{psec}$ in SL's and as such the above integrals sample a very narrow interval of time. The rateequation approximation exploits the rapid decay provided by $\gamma$ to remove slowly varying quantities from these integrations. From discussions in Sec. III variations in $\psi_{c}^{\dagger} \psi_{c}$ and $\psi_{v}^{\dagger} \psi_{v}$ can be separated into slowly and rapidly varying parts as follows:

$$
\begin{aligned}
& \psi_{c}^{\dagger} \psi_{c}=f_{c}+v_{c}, \\
& \psi_{v}^{\dagger} \psi_{v}=f_{v}+v_{v},
\end{aligned}
$$

where $f_{c}$ and $f_{v}$ are the slowly varying quasi-Fermioperators (varying on a nanosecond time scale) and $v_{c}$ and $v_{v}$ are the rapidly varying fluctuations caused by intraband thermalization. The time evolution of the Fermi operators is given by (3.41) and the time evolution of $v_{c}$ and $v_{v}$ follows immediately from (3.35) and (3.36),

$$
\begin{aligned}
& \dot{v}_{c}=-\frac{v_{c}}{\tau_{c}}+L_{c}(\mathbf{x}, \mathbf{k}, t), \\
& \dot{v}_{v}=-\frac{v_{v}}{\tau_{v}}+L_{v}(\mathbf{x}, \mathbf{k}, t),
\end{aligned}
$$

where we have treated $f_{c}$ and $f_{v}$ adiabatically and have also neglected the operator $Y$ in comparison to the intraband scattering terms. In addition to intraband scattering, multimode interactions can also cause rapid variations of the population (i.e., modal beating ${ }^{1}$ ). Provided that the intermodal beat frequency is smaller than the collisional dephasing rate (i.e., $\left|\omega_{l}-\omega_{m}\right|<\gamma$ ), however, the rate-equation approximation will remain valid. Nearly all lasing modes will satisfy this condition, because of the large $\gamma$ in SL's. Using the decomposition given in (4.2) and (4.3) and also separating the rapid optical variations in the operator $b_{l}^{\dagger}$, we can apply the rate-equation approximation to $(4.1)$ to yield

$$
\begin{aligned}
\psi_{c}^{\dagger} \psi_{v}= & -i \sum_{l} \frac{u_{l}(\mathbf{x}) q^{*}(\mathbf{k})}{\sqrt{2 \epsilon \hbar \omega_{l}}} \frac{\left(f_{c}-f_{v}\right) b_{l}^{\dagger}}{i\left[\bar{\omega}_{l}-\Omega(\mathbf{k})\right]+\gamma}-i \sum_{l} \frac{u_{l}(\mathbf{x}) q^{*}(\mathbf{k}) b_{l}^{\dagger}}{\sqrt{2 \epsilon \hbar \omega_{l}}} \int_{-\infty}^{t} d t^{\prime}\left(v_{c}-v_{v}\right) \exp \left(\left\{i\left[\Omega(\mathbf{k})-\bar{\omega}_{l}\right]-\gamma\right\}\left(t-t^{\prime}\right)\right) \\
& +\int_{-\infty}^{t} d t^{\prime} \Delta\left(\mathbf{x}, \mathbf{k}, t^{\prime}\right) \exp \left\{[i \Omega(\mathbf{k})-\gamma]\left(t-t^{\prime}\right)\right\}
\end{aligned}
$$

where $\bar{\omega}_{l}$ is the lasing frequency of the $l$ th mode (not necessarily equal to $\left.\omega_{l}\right)$. We have also replaced ( $\left.\mathbf{x}^{\prime}, \mathbf{k}^{\prime}\right)$ by $(\mathbf{x}, \mathbf{k})$ throughout this expression since any point in one of the phase spaces will not evolve significantly during the time interval $\gamma^{-1}$.

The operators $\psi_{c}^{\dagger} \psi_{v}$ and $\psi_{v}^{\dagger} \psi_{c}$ represent contributions to the active medium polarization caused by the electronradiation interaction between points in the respective conduction-band and valence-band phase spaces. Each term in (4.6) then represents a different contribution to polarization. The first term is the induced polarization, giving rise to a complex susceptibility which depends on the excitation of the active layer; the second term is an intraband scattering contribution to polarization (the so called "occupation fluctuation" contribution to polarization ${ }^{21}$ ); and the third term is the 
main contributor to quantum fluctuations in lasers. This term in conjunction with a contribution from optical-field vacuum fluctuations causes spontaneous emission fluctuations of the lasing modes.

The optical-field and carrier-density equations (3.33) and (3.41) are now rewritten using (4.6). We have

$$
\begin{aligned}
\dot{b}_{l}^{\dagger}= & {\left[i \omega_{l}-\frac{1}{2 \tau_{l}}\right] b_{l^{\prime}}^{\dagger}+i \int d \mathbf{x} u_{l}(\mathbf{x}) \sum_{m} \frac{\omega_{m}}{2 \mu^{2}} \chi\left(n, \bar{\omega}_{m}\right) b_{m}^{\dagger} u_{m}(\mathbf{x}) } \\
& +\sum_{m} \int d \mathbf{x} d \mathbf{k} \frac{|q(\mathbf{k})|^{2} u_{l}(\mathbf{x}) u_{m}(\mathbf{x})}{2 \epsilon \hbar \omega_{l}} b_{m}^{\dagger} \int_{-\infty}^{t} d t^{\prime}\left(v_{c}-v_{v}\right) \exp \left(\left\{i\left[\Omega(\mathbf{k})-\bar{\omega}_{m}\right]-\gamma\right\}\left(t-t^{\prime}\right)\right) \\
& +\frac{i}{\sqrt{2 \epsilon \hbar \omega_{l}}} \int d \mathbf{x} d \mathbf{k} d t^{\prime} u_{l}(\mathbf{x}) q(\mathbf{k}) \Delta\left(\mathbf{x}, \mathbf{k}, t^{\prime}\right) \exp \left\{[i \Omega(\mathbf{k})-\gamma]\left(t-t^{\prime}\right)\right\}+g_{l}^{\dagger}(t) \\
\partial_{t} n+\nabla \cdot \mathbf{J}= & -R+G+\sum_{l, m} \frac{\omega_{m}}{2 \mu^{2} i} u_{l}(\mathbf{x}) u_{m}(\mathbf{x})\left[\chi\left(n, \bar{\omega}_{m}\right) b_{m}^{\dagger} b_{l}-\mathbf{H . a .}\right] \\
& \quad-\sum_{l, m} \frac{u_{l}(\mathbf{x}) u_{m}(\mathbf{x})}{2 \epsilon \hbar \sqrt{\omega_{m} \omega_{l}}} \int d \mathbf{k} \int_{-\infty}^{t} d t^{\prime}|q(\mathbf{k})|^{2}\left(v_{c}-v_{v}\right)\left[b_{m}^{\dagger} b_{l} \exp \left(\left\{i\left[\Omega(\mathbf{k})-\bar{\omega}_{m}\right]-\gamma\right\}\left(t-t^{\prime}\right)\right)+\mathbf{H} . \mathbf{a} .\right] \\
& \quad-i \sum_{l} \frac{u_{l}(\mathbf{x})}{\sqrt{2 \epsilon \hbar \omega_{l}}} \int d \mathbf{k} \int_{-\infty}^{t} d t^{\prime}\left[q(\mathbf{k}) \Delta\left(\mathbf{x}, \mathbf{k}, t^{\prime}\right) b_{l} \exp \left\{[i \Omega(\mathbf{k})-\gamma]\left(t-t^{\prime}\right)\right\}-\text { H.a. }\right]+\int d \mathbf{k} L_{c}(\mathbf{x}, \mathbf{k}, t)
\end{aligned}
$$

where in writing these equations we have defined a local complex susceptibility operator as follows:

$$
\chi\left(n, \omega_{l}\right)=\frac{-i}{\epsilon_{0} \hbar \omega_{l}^{2}} \int d \mathbf{k} \frac{|q(\mathbf{k})|^{2}\left(f_{c}-f_{v}\right)}{i\left[\omega_{l}-\Omega(\mathbf{k})\right]+\gamma} .
$$

We take the complex susceptibility to be an explicit function of the carrier density $n$, rather than an equivalent representation in terms of either one of the electrochemical potentials.

Equations (4.4), (4.5), (4.7), and (4.8) represent the main result of our analysis. Although the rate-equation approximation has been invoked in the case of (4.7) and (4.8), the equations should remain exceedingly accurate owing to the smallness of $\gamma^{-1}$ in comparison to characteristic times of interest. Thus these equations can be used to study a variety of phenomena ranging from multimode interactions to the effect of carrier diffusion on optical fluctuations. Under circumstances where the rate-equation approximation does not hold the more general forms of these equations appearing in Sec. III can be used.

In this paper we have developed an operator formalism based on electronic wave packets which facilitates the treatment of local phenomena in semiconductors. In addition, an example of its use has been presented by developing local operator equations of motion for a semiconductor laser. In doing this we have rigorously incorporated the electro-optic interaction into the semiclassical picture of the Bloch electron, and have given conditions under which other quantum interactions may also be treated using this formalism. Although the analysis we have presented treats only the electronic system in a local fashion, it is clear that one can do likewise for the optical field by developing a space of optical wave packets. ${ }^{17}$ Such a formalism might prove to be useful for interpreting femtosecond-pulse propagation experiments.

\section{ACKNOWLEDGMENTS}

This work was supported by the Office of Naval Research, ITT Corporation, and Rockwell International. One author (K.J.V.) is grateful for financial support from IBM.
${ }^{1}$ W. E. Lamb, Jr., Phys. Rev. 134, 1429 (1964).

${ }^{2}$ H. Haken, Laser Theory (Springer, New York, 1983).

${ }^{3}$ H. Haug, Phys. Rev. 184, 338 (1969).

${ }^{4}$ H. Haug, Z. Phys. 200, 57 (1967).

${ }^{5}$ H. Haug and H. Haken, Z. Phys. 204, 262 (1967).

${ }^{6}$ H. Haug, Z. Phys. 206, 163 (1967).

${ }^{7}$ M. Yamada and Y. Suematsu, J. Appl. Phys. 52, 2653 (1981).

${ }^{8}$ See, for example, N. W. Ashcroft and N. D. Mermin, Solid State Physics (Holt-Rhinehart-Winston, Philadelphia, 1976).

${ }^{9}$ E. Ya. Glushko, Phys. Status Solidi B 114, 685 (1982).
${ }^{10}$ E. Wigner, Phys. Rev. 40, 749 (1932).

${ }^{11}$ Iu. L. Klimontovich, Zh. Eksp. Teor. Fiz. 33, 982 (1957) [Sov. Phys.-JETP 6, 753 (1957)].

${ }^{12}$ W. E. Brittin and W. R. Chappell, Rev. Mod. Phys. 34, 620 (1962).

${ }^{13}$ H. Frohlich, Physica 37, 215 (1967).

${ }^{14} \mathrm{~K}$. Vahala and A. Yariv (unpublished).

${ }^{15}$ C. Cohen-Tannoudji, B. Diu, and F. Laloe, Quantum Mechanics (Wiley, New York, 1977), Vol. 1, pp. 61-66.

${ }^{16}$ D. Stoler, Phys. Rev. D 1, 3217 (1970). 
${ }^{17}$ M. C. Teich, B. E. A. Saleh, and J. Perina, J. Opt. Soc. Am. B 1, 366 (1984).

18J. B. Moreno, J. Appl. Phys. 48, 4152 (1977).

${ }^{19}$ M. Lax, Phys. Rev. 145, 110 (1966).
${ }^{20}$ H. Haken and W. Weidlich, Z. Phys. 189, 1 (1966).

${ }^{21}$ K. Vahala and A. Yariv, Appl. Phys. Lett. 43, 140 (1983).

${ }^{22}$ R. Lang, J. Quantum Electron. QE-10, 825 (1974). 